\title{
The Effect of Microchimerism on Frequency and Severity of Organ Involve- ment in Iranian Scleroderma Patients
}

\author{
Sepideh Fathi Bitaraf ${ }^{1}$, Mohammadali Nazarinia ${ }^{2 *}$, Elmira Esmaeilzadeh ${ }^{1}$, Eskandar Kamali Sarvestani ${ }^{3}$, \\ Zohre Khodamoradi ${ }^{2}$ \\ ${ }^{1}$ Department of Internal Medicine, Division of Rheumatology, Shiraz University of Medical Sciences, Shiraz, Iran. \\ ${ }^{2}$ Geriatric Research Center, Shiraz University of Medical Sciences, Shiraz, Iran. ${ }^{3}$ Autoimmune Diseases Research \\ Center, Shiraz University of Medical Sciences, Shiraz, Iran
}

\begin{abstract}
Microchimerism is defined as the presence of non-self and circulating cells in a host. The current study aimed to assess the effect of microchimerism on scleroderma major organ involvements.

This cross-sectional study was conducted on 56 scleroderma patients registered in a tertiary rheumatology center of Shiraz University of Medical Sciences. Information on the patients' demographics and disease complications was gathered through a review of medical records. Skin score was applied to better assess skin thickening. High Resolution CT-scan as well as pulmonary function test (PFT) results were also used to investigate pulmonary involvement in patients. Y chromosome serum levels were measured using Phenol Chloroform Extraction protocol and following real-time PCR.

Fifty-six scleroderma patients with a mean age of $46 \pm 10$ years were recruited in this study $(58.9 \%$ with diffuse scleroderma and $41.07 \%$ with limited scleroderma). Other than skin thickening, the most common clinical presentation among the patients was interestitial lung disease (67.8\%). No significant difference was found between Y chromosome levels of patients with either lung, cardiac, renal, or gastrointestinal involvement and those who did not have these complications. Y chromosome serum levels based on the results of PFT were also shown to have no significant difference. Moreover, no association was demonstrated between serum $\mathrm{Y}$ chromosome and skin score.

The serum level of chromosome $\mathrm{Y}$ has no impact on the severity and frequency of major organ involvement in Iranian scleroderma patients.
\end{abstract}

Keywords: Autoimmune Disease, Systemic Sclerosis, Scleroderma, Interestitial Lung Disease, Microchimerism

\section{Introduction}

Systemic sclerosis (Scleroderma, SSc) is an autoimmune disease characterized by collagen deposition in the skin and multiple internal organs $[1,2]$. It has high incidence and prevalence in women of reproductive ages [2]. Study of the pathophysiology of this disease revealed that both genetic and environmental factors influence it. One recently proposed mechanism involved in the pathogenesis of $\mathrm{SSc}$ is microchimerism (Mc).

During pregnancy, the bidirectional flow of cells between mother and fetus occurs predominately from fetus to mother [3]. Most of these cells go through apoptosis and decrease in number following parturition; however, a small number of them can still be detected in maternal blood and tissue even decades after childbirth. This is known as Mc $[4,5]$. Whether or not these cells affect maternal health still remains open to question in the current literature $(6,7)$. Some studies have demonstrated the role of $\mathrm{Mc}$ in preg- nancy complications, wound healing, and multiple malignancies in mothers [3].

Recent research on the pathophysiology of autoimmune diseases, due to their female predominance, focused on the possible role of $\mathrm{Mc}$ in this group of disorders [1]. In previous studies, it was observed that women with scleroderma, rheumatoid arthritis, and Hashimoto's thyroiditis had a higher frequency of Mc compared with the normal population; consequently, it was concluded that this physiologic process can increase the risk of autoimmune diseases [8].

In their study on the association of Mc with clinical subsets of scleroderma, Sahin et al. concluded that Mc can shorten the elapsed time of disease development in scleroderma patients; however, it was determined to have no association with clinical presentations of the disease [9].

On the other hand, another investigation into the effects of number of parities and gender of children on the epidemiology and pathogenesis of scleroderma detected no sig-

Personal non-commercial use only.Rheumatology Research Journal. Copyright (C 2019. All rights reserved

*Corresponding Author: Mohammad Ali Nazarinia, Geriatric Research Center, Shiraz University of Medical Sciences, Shiraz, Iran, Email: nazariniam@gmail.com, Tel/Fax: (+98) 713-2337855, (+98) 713-2359847

Received: 02 Aguste 2018 ; Accepted: 03 February 2019 
nificant association, which put into debate the suggested role of $\mathrm{Mc}$ in the pathogenesis of scleroderma [10].

The mentioned controversies between previous studies about the effect of $\mathrm{Mc}$ on the pathogenesis and clinical course of autoimmune diseases such as scleroderma mandate further investigation on this topic. Moreover, due to the heterogeneity in prevalence and clinical presentations of SSc in different ethnicities and populations, it seems to be essential that more population-based studies be performed. Therefore, the current study aimed to investigate the effect of Mc on major organ involvement of SSc, including pulmonary, gastrointestinal, cardiac, and renal involvement.

\section{Materials and Methods}

A cross-sectional study was done on 56 married female scleroderma patients registered in a tertiary rheumatology center of Shiraz University of Medical Sciences. The patients' scleroderma was diagnosed based on LeRoy criteria [11].

\section{Inclusion Criteria}

Married female scleroderma patients with ages between 20-50 years were recruited in the present study. All patients had at least one male-gender child.

\section{Exclusion Criteria}

Patients with poor follow up or insufficient data in their medical records were excluded from the study. Patients with comorbid diseases such as diabetes, thyroid diseases, cardiovascular diseases, and hypertension, those who were cigarette smokers or opium addicts, and patients who had parietal complications including miscarriage, stillbirth, or death and those with twin siblings were also excluded from the study.

\section{Study Design}

\section{A. Demographic data and disease clinical presenta-} tions:

The patients' demographic information and major clinical presentations including skin, pulmonary, gastrointestinal, cardiac, and renal involvement were reviewed using their medical records.

To better assess skin thickening in patients, a skin score was estimated for each one [12]. Pulmonary involvement was also investigated using high-resolution computed topography (HRCT) in addition to pulmonary function test (PFT).

\section{B. Determining quantitative measure of $Y$ chromo-} some:

A 3-ml blood sample was obtained from each patient by an expert technician into $10 \mathrm{cc}$ BD Vacutainer EDTA tubes. Samples were then centrifuged at $3000 \mathrm{rpm}$ for 10 minutes at $4{ }^{\circ} \mathrm{C}$ and, approximately $1 \mathrm{cc}$ of buffy coat was collected and stored at $-80{ }^{\circ} \mathrm{C}$ for further processing.

DNA molecules were extracted using the phenol-chloroform extraction protocol [13]. They were then mixed with master mix (Cybergreen, Takara, Japan, 2018) dye and underwent real time PCR (Step One Real-Time PCR System, USA, applied biosystems) to measure Y chromosome levels.

\section{Statistical Analysis}

Data was entered into Statistical Analysis (SPSS) 19 software. Descriptive analysis was applied for reporting results on the prevalence of patients' demographic information and clinical presentations. The Mann-Whitney U test was also used to compare the $\mathrm{Y}$ chromosome levels between patients based on their major organ involvements. A p-value less than 0.05 was considered as statistically significant.

\section{Results}

Fifty-six married female scleroderma patients with a mean age at disease onset of $41 \pm 11$ years and a mean age of $46 \pm 10$ years at the beginning of the study were included in this study. Only $25 \%$ of participants had a positive family history for rheumatologic diseases. About 58.9\% and $41.07 \%$ of patients had diffuse and limited scleroderma, respectively. The demographic information and disease characteristics of participants are summarized in Table 1. The frequency of major organ involvement and clinical presentations are apparent from Table 2, which shows that skin involvement is the most frequent presentation; consequently, sclerodactyly and Raynaud were detected in all participants. However, only 33.9\% (19) of them had telangiectasia; $18(32.1 \%)$ patients had pitting at the fingertips, and $2(3.57 \%)$ patients had calcinosis. Moreover, $67.8 \%$ of participants had interstitial lung disease, and the most common symptom in patients with gastrointestinal involvement was dysphagia. Among 37 patients with gastrointestinal involvement, $9(24.3 \%)$ of them had diarrhea, $7(18.9 \%)$ had constipation, $4(10.8 \%)$ had vomiting, and $3(8.1 \%)$ patients had reflux. Four patients $(0.07 \%)$ in the current study had kidney involvement, and 16 (28.5\%) patients had cardiac involvement.

The serum levels of chromosome $\mathrm{Y}$ of patients were also compared based on the major clinical presentations, and the results are depicted in Table 3. The serum levels of chromosome $\mathrm{Y}$ were also compared with regard to the two major types of the disease, yet, no significant difference was found between patients with diffuse form $(0.037+-$ $0.05)$ and those with limited scleroderma $(0.078+-0.17)$ $(P$ value $=0.58)$. 
The Effect of Microchimerism on ...

Table 1. Patients' Demographic and Disease Characteristics

\begin{tabular}{lc}
\hline \multicolumn{1}{c}{ Patients' Characteristics } & Results \\
\hline Age of disease onset (mean \pm SD) & $41 \pm 11$ \\
Age at the time of study (mean \pm SD) & $46 \pm 10$ \\
Disease Duration (mean \pm SD) & $4 \pm 5$ \\
Positive family history of rheumatologic diseases (\%(NO./TOTAL NO.)) & $25(14 / 56)$ \\
Type of disease (\%(NO./TOTAL NO.)) & \\
1. Diffused & $58.9(33 / 56)$ \\
2. Limited & $41.07(23 / 56)$ \\
\hline
\end{tabular}

Table 2. Summery of patient's clinical presentations

\begin{tabular}{lc}
\hline \multicolumn{1}{c}{ Patients Major Clinical Presentations } & \%(NO./TOTAL NO.) \\
\hline Interstitial Lung Disease & $67.8(38 / 56)$ \\
Gastrointestinal Involvement & $66.07(37 / 56)$ \\
1. Dysphagia & $45.9(17 / 37)$ \\
2. Reflux & $8.10(3 / 37)$ \\
3. Diarrhea & $24.3(9 / 37)$ \\
4. Constipation & $18.9(7 / 37)$ \\
5. Vomiting & $10.8(4 / 37)$ \\
Skin Involvement & $100(56 / 56)$ \\
1. Sclerodactyly & $100(56 / 56)$ \\
2. Raynaud & $100(56 / 56)$ \\
3. Telangiectasia & $33.9(19 / 56)$ \\
4. Calcinosis & $3.57(2 / 56)$ \\
5. Digital Ulcer & $50(28 / 56)$ \\
6. Pitting at fingertips & $32.1(18 / 56)$ \\
Renal Involvement & $0.07(4 / 56)$ \\
Cardiac Involvement & $28.5(16 / 56)$ \\
\hline
\end{tabular}

Table 3. Comparing level of chromosome $\mathrm{Y}$ between patients regarding to their major clinical presentations.

\begin{tabular}{lccc}
\hline \multirow{2}{*}{ Organ Involvement } & \multicolumn{2}{c}{ Chromosome Y level (mean \pm SD ) } & \multirow{2}{*}{$P$ value } \\
\cline { 2 - 3 } & Patients with organ involvement & $\begin{array}{c}\text { Patients without organ } \\
\text { involvement }\end{array}$ & \\
\hline Lung Involvement (ILD) & $0.06 \pm 0.14$ & $0.03 \pm 0.07$ & 0.86 \\
Cardiac Involvement & $0.09 \pm 0.2$ & $0.03 \pm 0.06$ & 0.33 \\
Gastrointestinal involvement & $0.03 \pm 0.07$ & $0.08 \pm 0.1$ & 0.18 \\
Renal Involvement & $0.05 \pm 0.1$ & $0.05 \pm 0.1$ & 0.85 \\
\hline
\end{tabular}


No association was found between disease duration and skin score $(14.3 \pm 6.9)$ and $\mathrm{Y}$ chromosome levels $(0.054 \pm 0.12)(p=0.999)$.

Analysis showed that there was no significant association between lung involvement (ILD) and Y chromosome level ( $P$ value $=0.86)$, and no significant correlation was found between cardiovascular, gastrointestinal, or renal involvement and Y chromosome levels.

According to the PFT results, 42.9\% (24/56) had nor- mal PFT and 42.9\% (24/56) had restrictive lung disease. Comparing the $\mathrm{Y}$ chromosome levels between patients with restrictive lung disease results and those with normal PFT also showed no significant difference (normal PFT: $0.05 \pm 0.1$; restrictive lung disease: $0.39 \pm 0.05 ; P$ value $=$ $0.31)$.

Furthermore, the mean skin score in patients of this study was estimated to be $14.3 \pm 6.9$. No significant association was found between the level of Y chromosome and skin score in patients of this study $(P$ value $=0.96)$.

\section{Y chromosome}

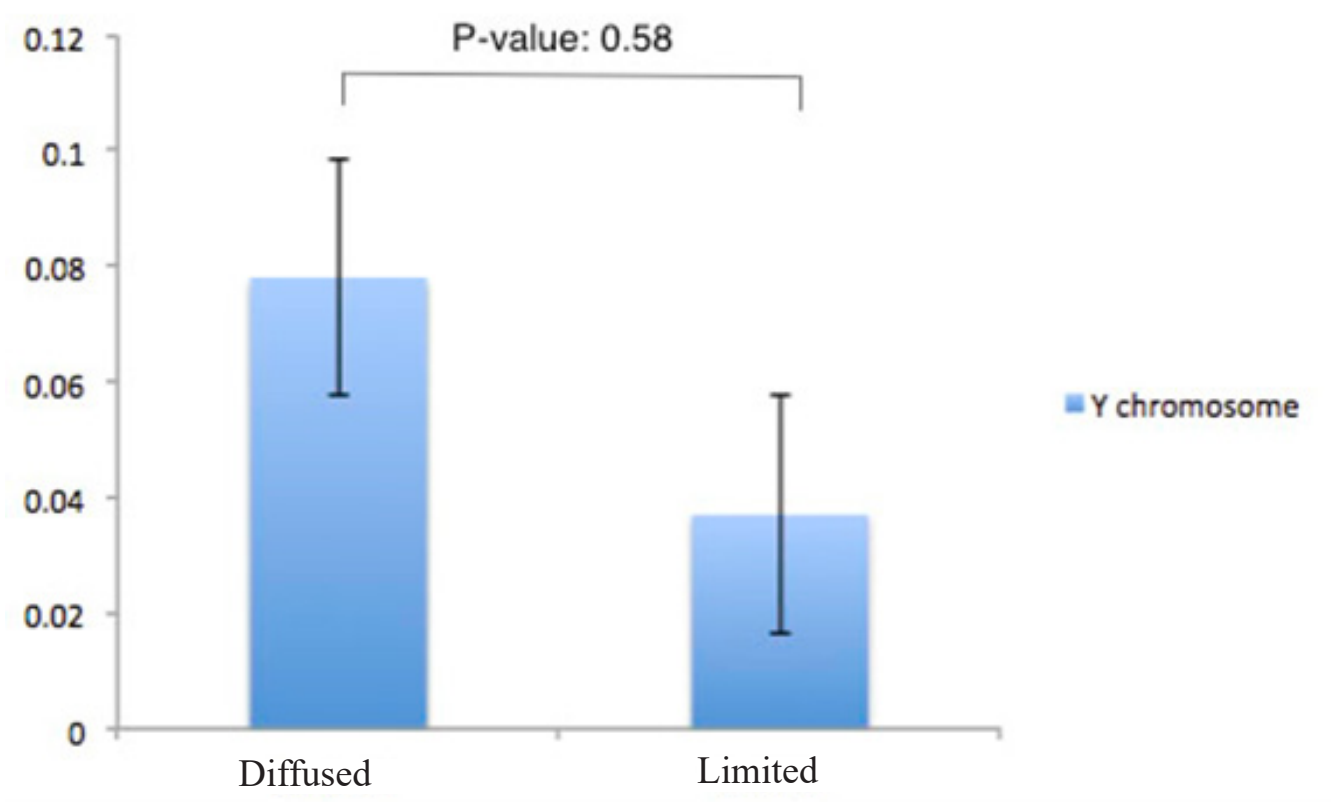

Figure 1. Comparing the serum level of chromosome $Y$ between diffused and limited type of scleroderma.

\section{Discussion}

Scleroderma, like most other autoimmune diseases, has a strong predilection for women with a mean age of $45-$ 54 years [14]. Other epidemiologic studies on scleroderma have also declared that the peak incidence of this disease in women is following childbearing age [15]. The mean age of patients in the current study was $46 \pm 10$ years at the time of the study, and at disease onset, it was $41 \pm 11$ years.

Microchimerism, as a physiologic finding in women following their pregnancies, was previously determined to have a role in the pathogenesis of autoimmune diseases, especially systemic sclerosis $[15,16]$. Some previous quantitative studies detected that the level of Mc was significantly higher in scleroderma women compared to normal healthy individuals [2]. Another study investigated a possible association between Mc and the limited form of SSc in addition to pulmonary fibrosis [17]. Some other studies have also supported the hypothesis on the role of Mc in the pathogenesis and organ involvement of SSc. A study by Johnson et al. on female scleroderma patients with at least one son detected that fetal cells can migrate form peripheral circulation into multiple organs. They suggested that it could support the role of Mc in the pathogenesis of SSc [18].

The current results revealed no significant difference in Y chromosome levels between scleroderma patients with either lung, cardiac, gastrointestinal, or renal involvement and ones without these complications. Moreover, using PFT, it was also determined that quantitative measures of Y chromosome do not differ between patients with restrictive patterns and ones with normal results. In addition, Y chromosome level was not associated with mean skin score, which was applied for assessing the severity of skin involvement in patients of the current study. No signifi- 
cant difference was found between the two major types of the disease. These findings, unlike most previous surveys, brought under question the role of $\mathrm{Mc}$ in the pathogenesis of scleroderma.

Some other studies can also be found which supported the current findings. A survey done on the effects of parity, family size, and gender of the children with the epidemiology and pathogenesis of scleroderma found no significant relationship [10]. Moreover, in evaluating the effect of prior pregnancies on scleroderma complications and death etiologies, it was manifested that nulliparous patients had earlier onset of the disease, more severe lung involvement, and a higher rate of death compared to multiparous ones [19].

These controversies regarding the role of $\mathrm{Mc}$ in the pathogenesis and clinical manifestations of scleroderma may be attributed to genetic variations between different ethnicities and cultures. In addition, the interval between the last pregnancy to lead to a son born in patients and the onset of their disease can also be another possible factor which may explain the differences between previous studies on the role of Mc in scleroderma. Nevertheless, further studies are needed to investigate these hypotheses.
The current study can be introduced as the first study to assess the effect of Mc on the frequency and severity of major clinical manifestations in Iranian scleroderma patients. However, it has also some limitations. One limitation of this study was its small sample size, which was inevitable due to the rarity of the disease and the presence of few female patients having a son. Furthermore, missing data, especially in patients' medical records, was another limitation which contributed to the small sample size. Nonetheless, efforts were made to minimize the amount of missing data on patients' clinical presentations by adding confirmatory tests such as HRCT, PFT, and skin score.

\section{Conclusion}

Unlike previous literature, it was concluded that the serum level of chromosome $\mathrm{Y}$ has no association with the severity and frequency of major organ involvement in Iranian scleroderma patients. This finding emphasized the possible role of genetic and ethnical variations in disease presentations, which mandates further regional investigations.

\section{Conflict of Interest}

The authors declare no conflicts of interest. 


\section{References}

1. Artlett CM. Microchimerism and scleroderma: an update. Curr Rheumatol Rep. 2003;5(2):154-9. doi: 10.1007/ s11926-003-0044-2.

2. Lambert NC, Lo YM, Erickson TD, Tylee TS, Guthrie KA, Furst DE, et al. Male microchimerism in healthy women and women with scleroderma: cells or circulating DNA? A quantitative answer. Blood. 2002;100(8):2845-51. doi: 10.1182/blood-2002-01-0295.

3. Boddy AM, Fortunato A, Wilson Sayres M, Aktipis A. Fetal microchimerism and maternal health: a review and evolutionary analysis of cooperation and conflict beyond the womb. Bioessays. 2015;37(10):1106-18. doi: 10.1002/ bies.201500059.

4. Bianchi DW, Zickwolf GK, Weil GJ, Sylvester S, DeMaria MA. Male fetal progenitor cells persist in maternal blood for as long as 27 years postpartum. Proc Nati Acad Sci $\boldsymbol{U} \boldsymbol{S}$ A. 1996;93(2):705-8. doi: 10.1073/pnas.93.2.705.

5. Bayes-Genis A, Bellosillo B, de la Calle O, Salido M, Roura S, Ristol FS, et al. Identification of male cardiomyocytes of extracardiac origin in the hearts of women with male progeny: male fetal cell microchimerism of the heart. J heart Lung Transplant. 2005;24(12):2179-83. doi: $10.1016 /$ j.healun.2005.06.003.

6. Mahmood U, O'Donoghue K. Microchimeric fetal cells play a role in maternal wound healing after pregnancy. Chimerism. 2014;5(2):40-52. doi: 10.4161/chim.28746.

7. Nassar D, Droitcourt C, Mathieu-d'Argent E, Kim MJ, Khosrotehrani K, Aractingi S. Fetal progenitor cells naturally transferred through pregnancy participate in inflammation and angiogenesis during wound healing. FASEB $\boldsymbol{J}$ 2012;26(1):149-57. doi: 10.1096/fj.11-180695.

8. Willer CJ, Sadovnick AD, Ebers GC. Microchimerism in autoimmunity and transplantation: potential relevance to multiple sclerosis. J Neuroimmunol. 2002;126(1-2):12633. doi: 10.1016/s0165-5728(02)00048-6.

9. Sahin A, Ozkan T, Turkcapar N, Kucuksahin O, Koksoy EB, Ozturk G, et al. Peripheral blood mononuclear cell microchimerism in Turkish female patients with systemic sclerosis. Mod Rheumatol. 2014;24(1):97-105. doi: 10.3109/14397595.2013.854052.

10. Russo PA, Lester S, Roberts-Thomson PJ. Systemic sclerosis, birth order and parity. Int J Rheum Dis. 2014;17(5):557-61. doi: 10.1111/1756-185x.12225.

11. LeRoy EC, Medsger TA, Jr. Criteria for the classification of early systemic sclerosis. J Rheumatol. 2001;28(7):15736.

12. Khanna D, Furst DE, Clements PJ, Allanore Y, Baron M, Czirjak L, et al. Standardization of the modified Rodnan skin score for use in clinical trials of systemic sclerosis. $\boldsymbol{J}$ Scleroderma Relat Disord. 2017;2(1):11-8. doi: 10.5301/ jsrd.5000231.

13. Barnett R, Larson G. A phenol-chloroform protocol for extracting DNA from ancient samples. Methods Mol Biol. 2012;840:13-9. doi: 10.1007/978-1-61779-516-9_2.
14. Medsger TA, Jr., Masi AT. The epidemiology of systemic sclerosis (scleroderma) among male U.S. veterans. J Chronic Dis. 1978;31(2):73-85. doi: 10.1016/00219681(78)90092-9.

15. Nelson JL. Microchimerism and the pathogenesis of systemic sclerosis. Curr Opin Rheumatol. 1998;10(6):56471. doi: 10.1097/00002281-199811000-00010.

16. Hadasik K, Bergler-Czop B, Brzezinska-Wcisto L. Fetal microchimerism in a pregnant woman and risk of autoimmune disease. Dematol Review. 2018;105(2):307-13. doi: $10.5114 / \mathrm{dr} .2018 .75586$.

17. Launay D, Hebbar M, Hatron PY, Michon-Pasturel U, Queyrel V, Hachulla E, et al. Relationship between parity and clinical and biological features in patients with systemic sclerosis. J Rheumatol. 2001;28(3):509-13.

18. Johnson KL, Nelson JL, Furst DE, McSweeney PA, Roberts DJ, Zhen DK, et al. Fetal cell microchimerism in tissue from multiple sites in women with systemic sclerosis. Arthritis Rheum. 2001;44(8):1848-54. doi:

19. Artlett CM, Rasheed M, Russo-Stieglitz KE, Sawaya $\mathrm{HH}$, Jimenez SA. Influence of prior pregnancies on disease course and cause of death in systemic sclerosis. Ann Rheum Dis. 2002;61(4):346-50. doi: 10.1136/ ard.61.4.346. 1002/1529-0131(200108)44:8<1848::aid$\operatorname{art323}>3.0 . \operatorname{co} ; 2-1$ 